\title{
Bleeding Aneurysms of the Basilar Artery. Direct Surgical Management in Four Cases*
}

\author{
Charles G. Drake
}

A few instances of direct or indirect surgical management of aneurysms on the vertebral-basilar system have been reported 2-7,9-12 (Fig. 1). Interesting is the fact that among 30 patients treated by direct methods there were only 4 deaths (including personal cases). Of the 8 patients treated indirectly by ligation of the vertebral artery, 1 died. Only 2 patients have been reported as disabled. Our interest in aneurysms on this circulation has increased with the reports of successful cases, and the knowledge that vertebral angiography will demonstrate a considerable proportion of the lesions responsible for spontaneous intra-cranial bleeding when bilateral carotid angiography is negative. Walton, ${ }^{15}$ following an extensive review of the literature, felt that 15 per cent of aneurysms were on the vertebralbasilar system. Bull ${ }^{13}$ reported that in 16 of 60 cases in which carotid angiography was negative, a vertebral angiogram demonstrated the lesion deemed to be responsible for the haemorrhage. Eight of these lesions were aneurysms, and 8 were angiomas. Two of the angiomas and 1 vertebral aneurysm were successfully excised. In London, Ontario, 30 vertebral angiograms have been done in the face of negative carotid angiography and lesions were found in 7 cases - 5 aneurysms and 2 angiomas. Thus it would appear that the site of bleeding will be discovered in 1 out of every 4 cases. In the event that the vertebral angiogram is normal, the patient and the family can be informed of the greatly lessened risk of recurrent bleeding.

In view of the limited surgical experience with these lesions, it seemed proper to present and discuss 4 cases in which a basilar aneurysm was attacked directly in urgent fashion because of repeated bleeding. Three patients were operated upon within 24 hours of the last haemorrhage, and the other 36 hours after.

The surgical method was similar in each case. Through dissection of a cadaver, it was seen that the upper one-third of the basilar artery and its bifurcation are exposed simply by an anterior subtemporal approach, through the tentorial opening into the inter-peduncular cistern. During induction of artificial hypothermia, a preliminary dissection of the neck was done in which both common carotid arteries were isolated under tapes and both vertebral arteries were exposed for three-quarters of an inch just below the carotid tubercle in the vertebral triangle. Subsequently, to facilitate final exposure of the aneurysm, bulldog clamps were placed on the carotid arteries and the vertebral arteries were occluded by the fingers of an assistant assigned for this purpose. This permitted total interruption of the cerebral circulation for brief periods. These usually were planned for 5 minutes at body temperatures between $26.0^{\circ} \mathrm{C}$. and $28.0^{\circ} \mathrm{C}$. and at least 5 minutes were allowed between interruptions.

With the patient in a semisupine position, a small temporal bone flap was turned down. The brain was made slack by intravenous 30 per cent urea and lumbar drainage of the cerebrospinal fluid. By retraction of the temporal lobe, the arachnoid was divided along the edge of the tentorium, exposing the entrance to the interpeduncular region between the carotid artery in front and the cerebral peduncle behind with the 3rd nerve angling across inferiorly. The anterior choroidal artery was held up gently by the tip of the retractor. The 4th nerve was tucked down below the tentorial edge. It was not necessary to divide the tentorial edge except in Case 4 where the bifurcation of the basilar artery seemed to be quite low. Brief periods of complete arrest of the cerebral circulation now were used to complete the dissection of the neck and adjacent fundus of the aneurysm, for the clot in the interpeduncular cistern has proved to be tough and stringy in each case. With a slack aneurysmal sac there is not the same danger of rupture and the surgeon can be more bold and swift with the exposure of the sac and placement of a clip. The neck of the aneurysms of the bifurcation was found by following the posterior cerebral artery medially and inferiorly as it curved around the peduncle. Only in Case 4 was it necessary to divide the posterior communicating artery. In Case 1, the artery was held up by the tip of the retractor and in Cases 2 and 3 , the artery was adherent to the fundus of the aneurysm and was occluded by the clip placed on the aneurysmal neck. The bone flaps were replaced in each case.

Case 1. A.N., a 50-year-old farmer, was well until Dec. 21, 1958, when he spoke incoherently to his sister on the telephone. He was admitted to his local hospital complaining of headache, diplopia and fatigue. During the following week he improved on rest in bed and sedation although the diplopia persisted intermittently. On December 29, he suddenly collapsed and was unconscious for 10 minutes, After transfer to the Neurosurgical Service he complained of severe frontal headache, diplopia and a desire to sleep and was amnesic for the week's events.

Examination. He was clammy, had a stiff neck, but there were no other abnormal neurological findings. The bloody spinal fluid was under a pressure of 580 .

Bilateral carotid angiography did not disclose the source of the haemorrhage. On January 3, a right submastoid vertebral angiogram demonstrated the presence of a bilocular saccular aneurysm arising from the basilar artery at the level of origin of the superior cerebellar arteries (Fig. 2a). The separation of the artery from the clivus suggested the presence of local subdural clot.

Course. Meanwhile he had improved but on the morning of January 7 he complained to the nurse of pain beginning in his feet and spreading to his head. This was followed by loss of consciousness for several minutes with fixed pupils. Two hours later he was still drowsy and confused with early choking of the optic discs and a haemorrhage in the right fundus. He appeared to have paresis of upward gaze and a slight divergent squint. His family was now ready to accept the risk of operation.

Operation. A right temporal bone flap was turned down to uncover a brain now slack after $210 \mathrm{cc}$. of 30 per cent solution of urea had been given intravenously. The body temperature was $26.5^{\circ} \mathrm{C}$. Through a subtemporal exposure, the arachnoid was divided along the edge of the tentorium. The 4th nerve was protected with a patty and the anterior choroidal and posterior communicating arteries were held up gently with the tip of the retractor. It was not necessary to divide the tentorium. There seemed to be an extra layer of dense arachnoid covering the clot in the interpeduncular cistern, which was divided piecemeal with a blunt hook. An assistant now occluded each

* Presented in part to the American Academy of Neurological Surgery, Pebble Beach, California, October, 1959.

Reprinted with permission from: Journal of Neurosurgery 1961, Vol. XVIII, No.2 Pages 230-238 


\section{BLEEDING ANEURYSMS OF BASILAR ARTERY}

INTRACRANIAL HEMORRHAGE WITH VERTEBRAL-BASILAR ANEURYSMS SITES IN CASES OPERATED UPON

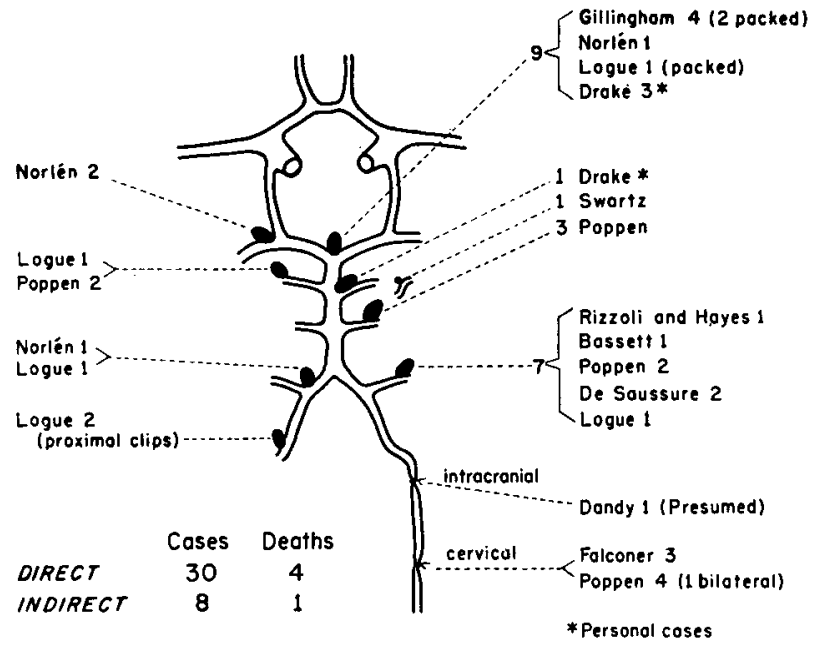

Fig. 1. Sites of vertebral-basilar aneurysms with intracranial haemorrhage in patients operated upon.
Examination. Bilateral carotid and right vertebral angiography were considered to be negative.

Course. The third haemorrhage occurred 10 days later while she was on the bedpan. She was drowsy and incontinent for 4 days with no evidence of focal disturbance of cerebral function. Recovery seemed complete after 4 weeks of total rest in bed. A few minutes after sitting up for the first time she again became deeply unconscious for 12 hours. Again bilateral carotid angiography was negative, but a vertebral arteriogram revealed a narrow saccular aneurysm arising from the bifurcation of the basilar artery and projecting anteriorly (Fig. 3a). A review of the previous angiograms failed to reveal any evidence of this lesion and there was no adjacent arterial spasm. A few hours later she could be roused enough to determine that there were bilateral 6th nerve palsy and paresis of upward gaze. There were no retinal haemorrhages or hemiparesis, but she seemed unable to speak.

Operation was begun 20 hours after this fourth haemorrhage. Artificial hypothermia to $26^{\circ} \mathrm{C}$. was induced and intravenous urea and lumbar drainage were used to make the brain slack. Two periods of total interruption of the circulation lasting 5 and 8 minutes were needed to remove the surrounding clot and place a Mayfield clip across the neck of the aneurysm, including the small posterior communicating artery which was adherent to the fundus and neck of the sac (Fig. 3b). The bone flap was replaced after the dural closure and a tracheotomy was done.

Course. Parenteral papaverine therapy was begun. She remained unresponsive for 5 days, then improved gradually but she did not become ambulatory for 6 weeks. A postoperative vertebral arteriogram 4 weeks after operation showed the aneurysm to be obliterated (Fig. 3c). Her course in hospital was marred by incontinence, a Korsakoff-like state and cortical blindness. She was discharged $2 \frac{1}{2}$ months after operation, continent and ambulatory. vertebral artery with a forefinger while the common carotid arteries were occluded with bulldog clamps. Two periods of complete interruption of the cerebral circulation, lasting 5 and 6 minutes respectively, with a 5-minute interval, allowed the exposure of the aneurysm to be completed. The clot in the interpeduncular cistern was sucked away carefully so that the aneurysm was exposed completely and the origin from the basilar artery could be seen by retracting the 3rd nerve caudally with a blunt hook (Fig. 2b). The circulation was interrupted for a final 8 minutes. During this time a few more strands of arachnoid were divided in order to increase the opening between the retractor and the 3 rd nerve. Actually the 3rd nerve had to be held down with a blunt hook using a little more force than one would desire in order to get the clipapplying forceps through the opening. Then, with the neck of the aneurysm in clear view, a wide Olivecrona clip was applied firmly, flush with the basilar artery. Hammered muscle was packed about the aneurysm. The dura mater was closed and the bone flap was replaced.

Course. After transfer to the recovery room, and while still hypothermic $\left(29^{\circ} \mathrm{C}\right.$.), a period of profound hypotension ensued lasting 15 to 20 minutes. Normal levels of blood pressure were sustained with Laevophed which was discontinued after 10 hours. Recovery of consciousness was prompt but he was confused intermittently and incontinent during the first 2 weeks. A right 3rd nerve palsy with only slight enlargement of the pupil persisted for 1 week, then rapidly improved. Vertebral angiography 2 weeks after operation showed the aneurysm to be obliterated (Fig.2c). He was discharged at the end of the 3rd week, having become ambulatory and showing subsiding papilloedema and paresis of upward gaze. The diplopia had disappeared and there were no signs of 3rd nerve palsy. At review 1 year later, the patient was working and neurological findings were normal.

Case 2. Mrs. K., a 45-year-old obese Dutch housewife under therapy for hypertension (170/110), was admitted on Oct. 4, 1959 after two episodes of bleeding in the preceding 12 hours. Loss of consciousness had been brief and associated with bilateral pin-point pupils and extensor plantar responses. Recovery was rapid and, apart from headache and stiff neck, there were no significant neurological changes.
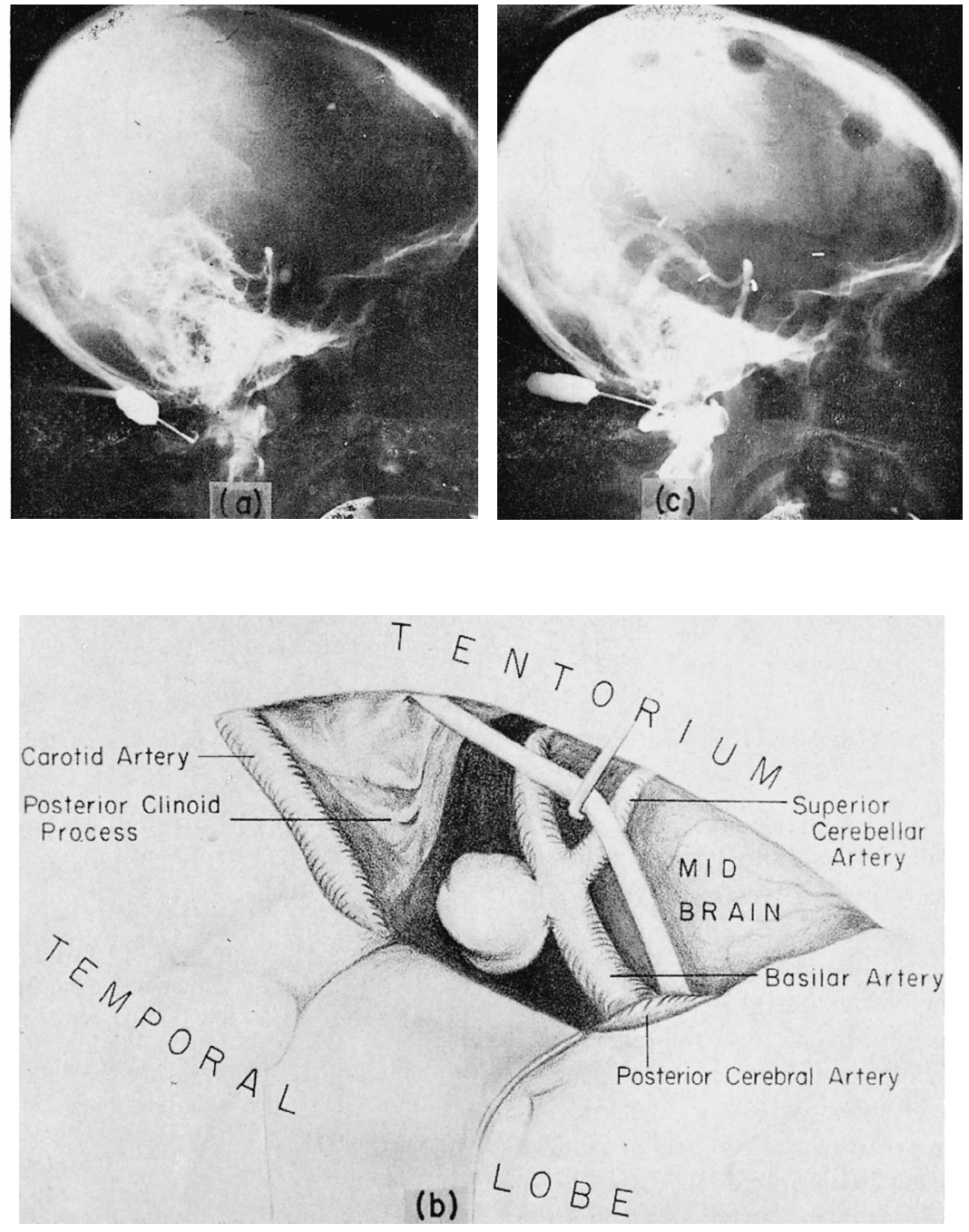

Fig. 2. Case 1. 

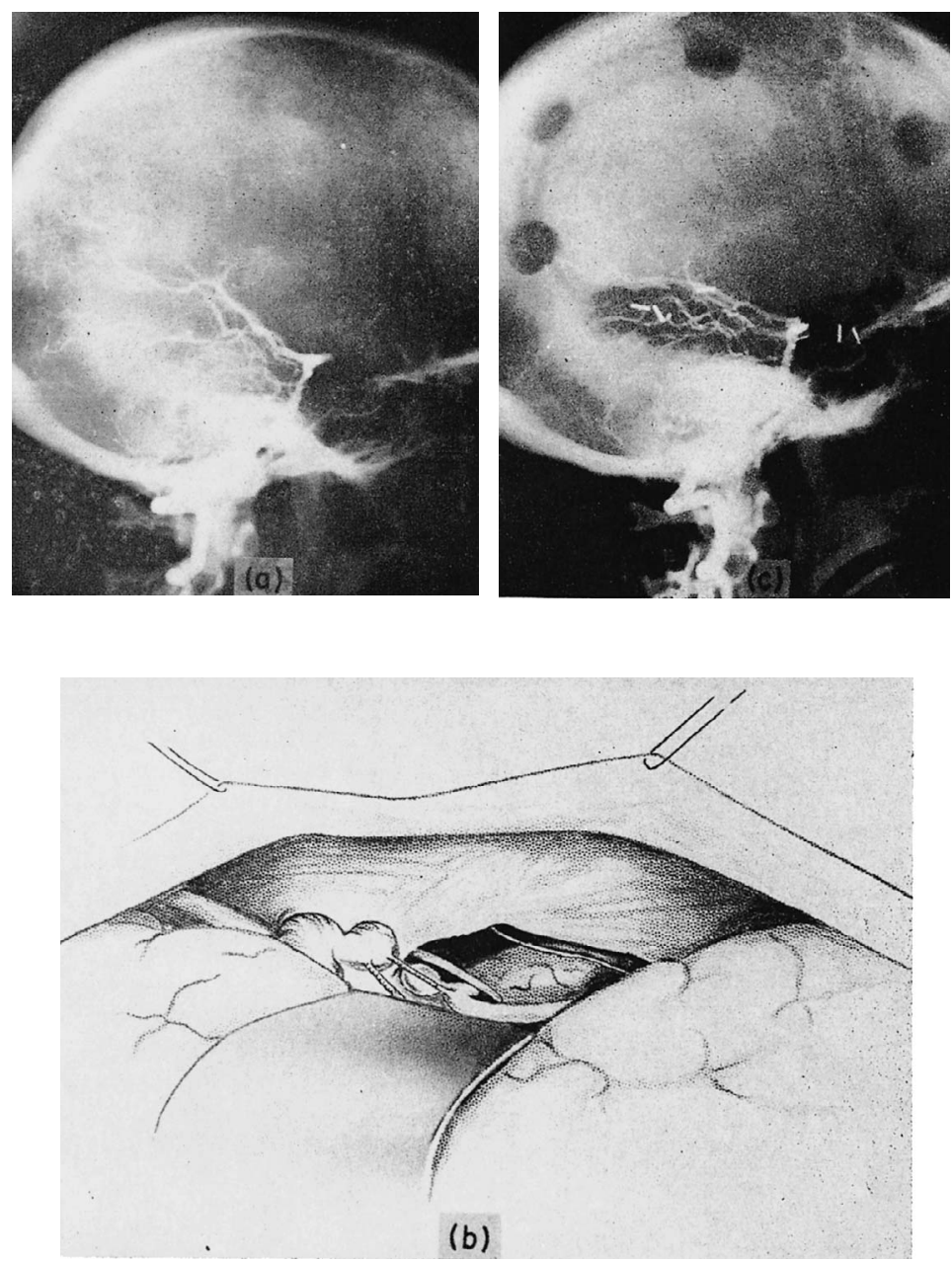

Fig. 3. Case 2.

Although she became mentally well, resolution of the bilateral central visual-field defect was slow. After 8 months, useful vision had returned to the point of reading large newspaper print. Her family was delighted with her recovery.

Comment. The amaurosis was not explained clearly. Both posterior cerebral arteries filled well, discounting the possibility of their occlusion by the clip or that a thrombus had formed or had been extruded into the distal part of the basilar artery. The periods of total interruption of the carotid and vertebral arteries were hardly long enough at $26^{\circ} \mathrm{C}$. to induce cortical necrosis. As there was no known period of postoperative hypotension, the most likely explanation of the bilateral occipital cortical infarction was intense postoperative spasm of the distal basilar or posterior cerebral arteries.

Case 3. D.C., a 45-year-old spinster, was admitted on Dec. 19, 1959 for the investigation of a left homonymous hemianopsia first noticed by the patient 6 months before.

Examination. Right carotid angiography was negative and an air encephalogram seemed normal except for poor filling of the 3rd ventricle. At this point, she admitted to having been in a hospital for "subarachnoid haemorrhage" 14 years before.

Course. Vertebral angiography was refused and she was discharged.

Four nights later she complained of severe headache but seemed better in the morning. A neighbour found her unconscious $1 / 2$ hour later. She was readmitted in deep coma with a left hemiplegia and a fixed dilated right pupil. Her sole response was a feeble picking movement with the right hand. Immediate vertebral angiography revealed the presence of a large aneurysm arising from the termination of the basilar artery and projecting forward and outwards into the right temporal lobe (Fig. 4). After some debate it was felt that an operation was warranted because of the undoubted presence of a clot.

Operation. During induction of hypothermia to $28^{\circ} \mathrm{C}$. dissection of the neck was done and a right temporal bone flap was turned down. The brain slackened off following removal of a diffuse, fresh subdural clot. The subtemporal clot was continuous with another mass of fresh clot in the depths of the temporal lobe around the lateral aspect of the aneurysm. The 3 rd nerve was separated from the sac below and then, with $7 \frac{1}{2}$ minutes of carotid and vertebral occlusion in the neck, the dissection of the neck of the aneurysm was completed and a long Mayfield clip was applied (Fig. 4b). The neck seemed much wider than indicated by the arteriogram and it was necessary to compress the adjacent fundus with bayonet forceps in order to apply the clip. The bone flap was replaced and a tracheotomy was done.

Course. Artificial hypothermia $\left(28^{\circ}-30^{\circ} \mathrm{C}\right.$.) was continued and she was able to open her eyes and move the left leg well and the left arm feebly. Death occurred on the 3rd postoperative day following the gradual onset of hyperventilation with hypotension which did not respond to vasopressor agents and intravenous urea.

Autopsy disclosed that the clip failed to completely occlude the aneurysmal neck, for a small probe could be passed beyond the far edge of the clip. There was no evidence of postoperative bleeding or thrombosis in the major vessels. A large amount of clot was found below the tentorium around the pons and medulla (Fig. 4c).

The fourth patient was seen and operated upon in conjunction with Dr. Ernest Sachs, Jr., at the Mary Hitchcock Memorial Hospital in Hanover, New Hampshire. I am indebted to Dr. Sachs for permission to report on this patient. 

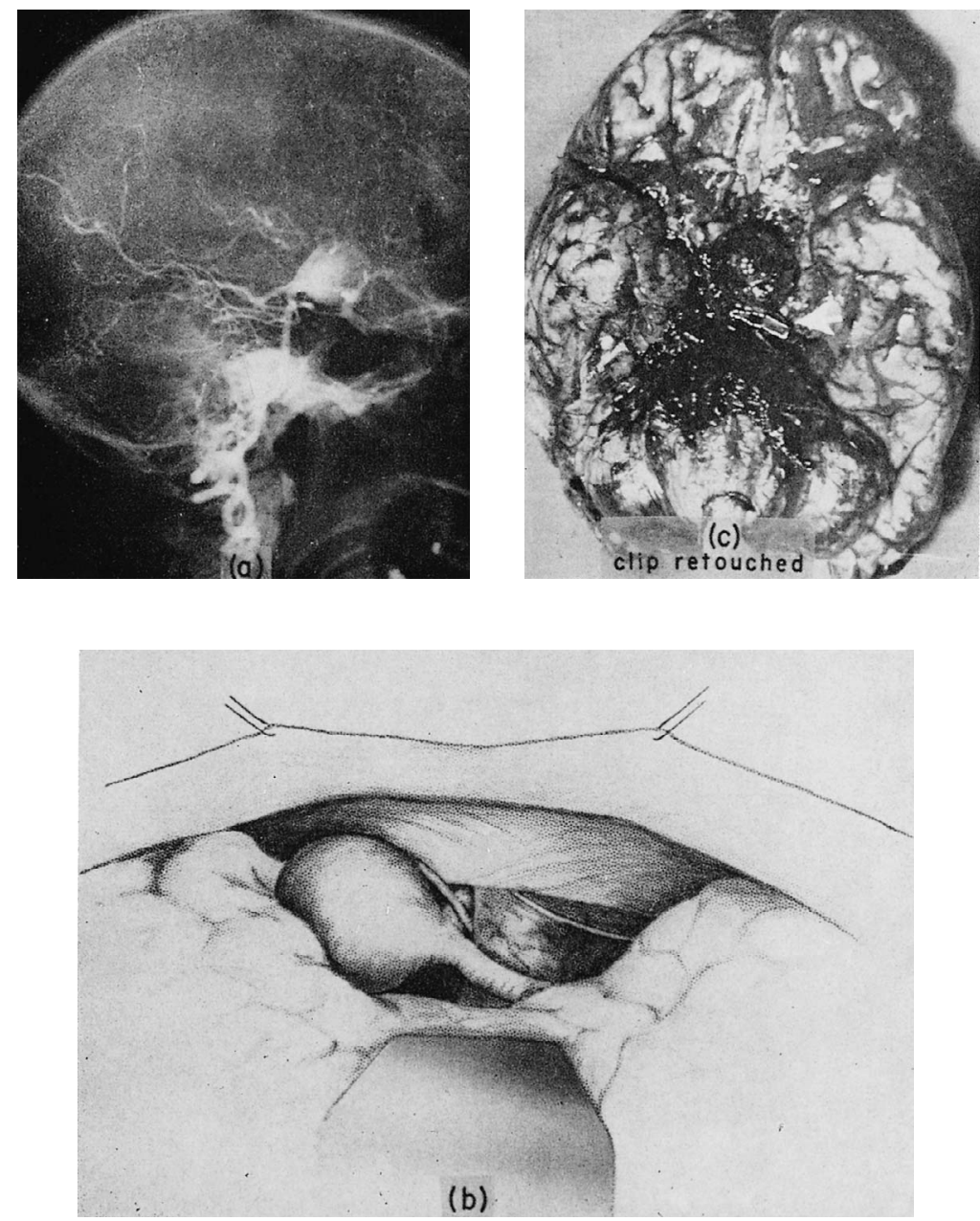

Fig. 4. Case 3.

Case 4. P.P., a 52-year-old woman, had her first and most severe subarachnoid haemorrhage on Dec. 30, 1959, and was admitted in a semiconscious state with rigid neck and bilateral extensor plantar responses.

Examination. Immediate bilateral carotid angiography was negative. The patient recovered quickly and vertebral arteriography 5 days later revealed a large saccular aneurysm arising from the termination of the basilar artery, projecting posteriorly and upwards (Fig. 5a).

Course. The following night, a second milder haemorrhage occurred with headache but no focal disturbance of cerebral function. The spinal fluid again showed evidence of fresh bleeding.

Operation was carried out 36 hours after the last haemorrhage under hypothermia with intravenous urea and lumbar drainage of the cerebrospinal fluid. The arachnoid was very dense in the interpeduncular fossa and was divided piecemeal with a blunt hook. The small posterior communicating artery was coagulated and divided at its middle and the posterior stump was used to retract the posterior cerebral artery away from the base of the aneurysm. Finally with two periods ( 3 and 5 minutes) of total arrest of cerebral circulation at $26.5^{\circ} \mathrm{C}$. excellent exposure of the base and neck of the aneurysm was obtained (Fig. 5b). At this point, although the body temperature had drifted lower than desired, the patient was moving around enough to warrant the injection of more relaxant (Flaxedil). With the aneurysm now exposed for clipping, the condition of the patient suddenly deteriorated and cardiac standstill occurred without ventricular fibrillation. Cardiac massage maintained the circulation until the electrical pacemaker took over. A long Mayfield clip was applied to the neck of the aneurysm and the wounds of the head and neck were closed. Normal cardiac rhythm was never established.

At autopsy the clip completely occluded the aneurysmal sac (Fig. 5c). Examination of the heart and brain disclosed no reason for the cardiac arrest.
Comment. Subsequent discussion of the disappointing outcome in this case with Lougheed and Heimbecker of Toronto pointed out that hearts at standstill at low temperatures ordinarily do not recover their rhythm properly until the body temperature is raised to about $30^{\circ} \mathrm{C}$. Perseverance along this line might well have spared this patient's life.

\section{Discussion}

Hamby ${ }^{8}$ described three varieties of aneurysm on the vertebral and basilar arteries and their branches: (A) Large tortuous, dilated "S"-shaped, arteriosclerotic vertebral and basilar arteries. (B) Spherical basilar or vertebral aneurysms. (C) Small saccular aneurysms with subarachnoid haemorrhage.

He considered that groups A and B presented chiefly signs of focal disturbance of brain-stem or cranial-nerve function and that considering their character little could be accomplished, especially when the lesion was in continuity with the main blood stream. Indirect attack by ligation of the vertebral artery was first practised by Dandy, ${ }^{3}$ and Falconer ${ }^{6}$ has mentioned 3 further cases with 1 death. Logue ${ }^{5}$ used proximal clips on two of his three vertebral aneurysms and stated that, contrary to prevailing views (Sweet ${ }^{1}$ ), the flow of blood in the vessel was reversed and the pressure was reduced profoundly. Hamby expressed a little more hope for a few of the large spherical lesions, and particularly the small saccular aneurysms. The former might be clipped or incised and packed as mentioned by Tönnis. ${ }^{14}$ Case 3 was representative of group B. This large aneurysm proved formidable at 


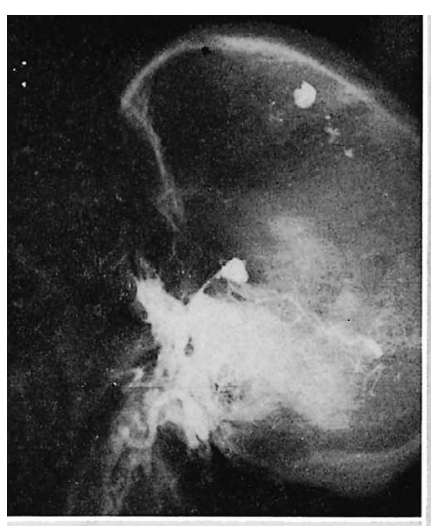

(a)

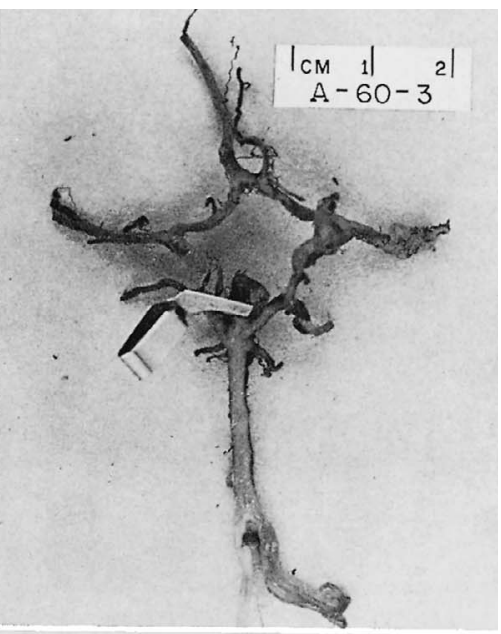

(c)

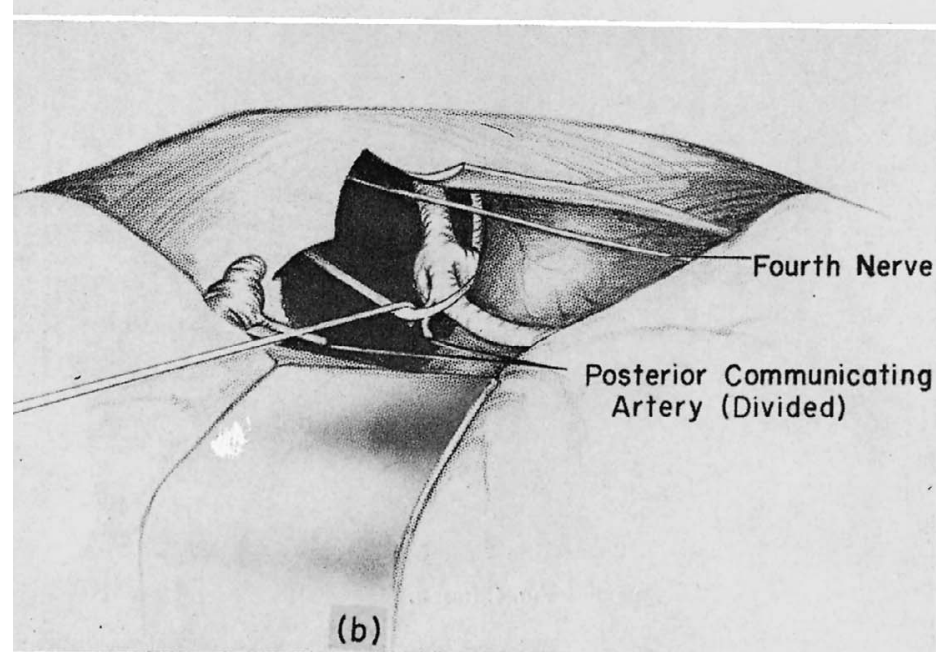

Fig. 5. Case 4. operation, for our longest clip just failed to occlude the neck completely. Removal of acute subdural and temporal lobe clot resulted in temporary improvement. Death occurred from brain-stem failure presumably because the thick layer of clot below the tentorium and surrounding the pons and medulla was not removed.

A number of these large sessile sacs may be handled best by reinforcement with muscle or plastic if the fundus can be exposed safely after removal of clot. Certainly in many of these lesions the better part of judgement will be retreat without specific management rather than risk rupture of the sac or compromise of the basilar circulation by an attempt to occlude a broad aneurysmal neck. In the event that the neck can be occluded safely, the fundus of a large sac probably should be collapsed by aspiration to remove the spaceoccupying effect in this confined region.

Several small, saccular aneurysms on the vertebral and the branches of the vertebral and basilar arteries have been clipped or excised successfully. Saccular aneurysms of the basilar artery have remained aloof because of the presumed limitations of accessibility and the fear of the consequences of changes in the basilar circulation. Actually the smaller, terminal basilar aneurysms are exposed simply by the subtemporal route used in these cases. The base and neck of the sac are not buried in cerebral substance but lie freely exposed in the interpeduncular subarachnoid cistern. Division of the tentorium exposes the lower basilar trunk although it is partially obscured by the 5th, 7th and 8th cranial nerves.

The exposure and application of a clip seemed quite straightforward in our cases representing this group (Cases 1, 2, and 4). Recovery was prompt in Case 1 and the patient has remained well for 1 year. Retraction of the 3rd nerve in order to gain access to the interpeduncular cistern may produce a paresis of this nerve. However in this case, in which the retraction necessary seemed more forceful than one would desire, the paresis was temporary and normal function of the 3rd nerve had returned after 2 weeks. The result in Case 2, very ill after a fourth haemorrhage, was marred by postoperative central-field blindness, the result of calcarine infarction, as yet unexplained. Case 3 , in extremis from massive bleeding from a large aneurysm, was probably irretrievable under any circumstances, yet an attempt at salvage surgery seemed to be warranted.

The surgical treatment of these aneurysms will undoubtedly develop in some degree and it would appear that in certain instances in which expectancy of life is poor, as with repeated haemorrhages, a direct attack is justifiable. The route used in these cases, through the middle fossa into the mouth of the incisura, will allow visualization and obliteration of certain small saccular aneurysms arising from the rostral portion of the basilar trunk and its bifurcation, as well as the proximal portion of the posterior cerebral artery and the superior cerebellar artery. 
The writer wishes to acknowledge with sincere thanks the efforts of Mrs. Margaret Corrin in the preparation of the illustrations.

\section{ADDENDUM}

Following the submission of this manuscript a fifth saccular aneurysm of the basilar bifurcation was occluded successfully at the Western Hospital, Toronto, through the courtesy of Dr. Ross Fleming.

On Dec. 18, 1960, a 48-year-old man (H.C.) suffered his first haemorrhage and was admitted in a drowsy state with bilateral extensor plantar responses and bloody spinal fluid. Bilateral carotid angiography was negative, but a vertebral arteriogram demonstrated a small saccular aneurysm arising and projecting forwards and upwards from the bifurcation of the basilar artery. He recovered quickly and it was decided to delay operation for 7 days. Unfortunately on December 26 right hemiparesis and dysphasia developed gradually without marked change in consciousness.

Left carotid angiography again was normal and as he seemed to be deteriorating, presumably from recurrent bleeding, operation was carried out on December 27 under hypothermia $\left(28^{\circ} \mathrm{C}\right.$.) with urea. Three brief periods $\left(3,5\right.$ and $1 \frac{1}{2} \mathrm{~min}$.) of complete occlusion of the vertebral and carotid arteries were used to complete the dissection of the aneurysm and occlusion of its neck with a McKenzie clip.

The postoperative course was encouraging. A 3rd nerve palsy improved as well as the hemiparesis and dysphasia. A vertebral arteriogram showed the aneurysm to be obliterated completely. However, after 3 weeks he was not as responsive, with high spinal fluid pressures. Ventriculography showed a moderate communicating hydrocephalus and a lumboperitoneal shunt done on February 4 has been followed by remarkable improvement. The hemiparesis and dysphasia have cleared but the 3rd nerve palsy is resolving more slowly.

\section{REFERENCES}

1. Bakay L and Sweet WH. Intra-arterial pressures in the neck and brain. Late changes after carotid closure, acute measurements after vertebral closure. J Neurosurg 1953;10:353-359.

2. Bassett RC. Intracranial aneurysms. I. Some clinical observations concerning their development. J Neurosurg 1949;6:216-221.

3. Dandy WE. Intracranial arterial aneurysms. Ithaca, NY. Comstock Publishing Co. Inc 1944, viii, 147pp.

4. DeSaussure RL, Hunter SE and Robertson JT. Saccular aneurysms of the posterior fossa. J Neurosurg 1958;15:385-391.

5. Dimsdale H, and Logue V. Ruptured posterior fossa aneurysms and their surgical treatment. J Neurol Neurosurg Psychiat 1959;n.s.22:202-217.

6. Falconer MA. Surgical treatment of spontaneous intracranial haemorrhage. Brit Med J 1958;1:790-792

7. Gillingham FJ. The management of ruptured intracranial aneurysm. Hunterian Lecture delivered at the Royal College of Surgeons of England on 24th January, 1957. Ann Roy Coll Surg Engl 1958;23:89-117.

8. Hamby WB. Intracranial aneurysms. Springfield, Ill. Charles C. Thomas 1952, xxi, 564pp.

9. Norlén G. Personal communication, Sept. 1959.

10. Poppen JL. Vascular surgery of the posterior fossa. Proc Congr Neurol Surg 1959;6:198-209.

11. Rizzoli HV and Hayes GJ. Congenital berry aneurysm of the posterior fossa. Case report with successful operative excision. J Neurosurg 1953; 10:550-551.

12. Schwartz HG. Arterial aneurysm of the posterior fossa. J Neurosurg 1948;5:312-316.

13. Spatz EL and Bull JWD. Vertebral arteriography in the study of subarachnoid haemorrhage. J Neurosurg 1957;14:543-547.

14. Tönnis W. Zur Behandlung der intrakranieller Aneurysmen. Arch Klin Chir 1937; 189:474-476.

15. Walton JN. Subarachnoid haemorrhage. Edinburgh \& London: E. \& S. Livingstone Ltd. 1956,xv,350pp. 